CLNS 93/1263

\title{
Particle-Field Duality and Form Factors from Vertex Operators
}

\author{
Costas Efthimiou and André LeClair \\ Newman Laboratory \\ Cornell University \\ Ithaca, NY 14853
}

\begin{abstract}
Using a duality between the space of particles and the space of fields, we show how one can compute form factors directly in the space of fields. This introduces the notion of vertex operators, and form factors are vacuum expectation values of such vertex operators in the space of fields. The vertex operators can be constructed explicitly in radial quantization. Furthermore, these vertex operators can be exactly bosonized in momentum space. We develop these ideas by studying the free-fermion point of the sine-Gordon theory, and use this scheme to compute some form-factors of some non-free fields in the sine-Gordon theory. This work further clarifies earlier work of one of the authors, and extends it to include the periodic sector.
\end{abstract}

$12 / 93$ 


\section{Introduction}

For relativistic quantum field theories with a massive particle spectrum, the main dynamical properties one is interested in are the S-matrix, the form factors of all local fields, and the Green's functions of these fields. For the integrable quantum field theories in 2 space-time dimensions, some of these properties have been computed exactly. The algebraic structures that characterize the S-matrices are well-known [1], and have been used to compute them for a wide variety of models. Bootstrap axioms satisfied by the form factors have been formulated[2] [3]. Important progress in solving the bootstrap for the multiparticle form factors was made by Smirnov[ [4] [3], where he computed exactly the form factors of certain basic fields, such as the energy-momentum tensor and global conserved currents, in the sine-Gordon (SG) model, $S U(N)$ Thirring model, and $O(3)$ non-linear sigma model.

It is of interest to develop a more algebraic framework for the computation of form factors, with the aim of constructing the solutions for the complete set of fields in an

efficient manner. A deeper understanding of such algebraic structures is likely to facilitate generalizations to other models, and could lead to some much-needed new approaches to the problem of computing Green's functions. In the works [5] [6] two new approaches to the computation of form factors were proposed. In [5], structures in radial quantization were used to construct form factors explicitly as vacuum expectations of vertex operators in momentum space. The ideas underlying the construction were developed at the free fermion point of the sine-Gordon theory. This case is not completely trivial, since there are fields in the sine-Gordon theory, such as $\exp ( \pm i \phi / 2)$, with non-trivial form factors and Green's functions since these fields are non-local in terms of the fermion fields. In Lukyanov's approach [6], the form factors are constructed as traces over auxiliary Fockmodules. The original motivation behind his construction came from the work [7], where the necessary properties of these traces were understood in the context of lattice models. The significant differences between the approaches in [5] and [6] is explained by the fact that whereas in [5] radial quantization is used, the construction in [6] appears to correspond to angular quantization. To see this, define the usual Euclidean light-cone and polar coordinates as follows

$$
z=(t+i x) / 2=\frac{r}{2} \exp (i \varphi), \quad \bar{z}=(t-i x) / 2=\frac{r}{2} \exp (-i \varphi) .
$$


In radial quantization $r$ is declared as the 'time', whereas in angular quantization $\varphi$ is the 'time'. In angular quantization, since the Lorentz boost operator $L$ generates shifts in $\varphi$, it is the Hamiltonian. Then, functional integrals can be represented as traces:

$$
\langle 0|\mathcal{O}| 0\rangle=\frac{\int D \Phi e^{-S} \mathcal{O}}{\int D \Phi e^{-S}}=\frac{\operatorname{Tr}\left(e^{2 \pi i L} \mathcal{O}\right)}{\operatorname{Tr}\left(e^{2 \pi i L}\right)}
$$

The $2 \pi i$ constant in the factor $\exp (2 \pi i L)$ is fixed by the $2 \pi$ length of the 'time' $\varphi$. In [7] [6], the latter constant was fixed by imposing the right symmetry properties of the form factors expressed as these traces.

In this paper, we elaborate further on the construction in [5]. We explain quite generally how the computation of form factors can be carried out in the space of fields, and how this is dual to the usual computation in the space of particles. This leads to a precise notion of vertex operators and their utility for computing form factors. We continue to study the free fermion point of the sine-Gordon theory, since our interests at this stage are mainly to understand more completely the physical features that make this new approach possible. With this understanding, we treat also the periodic sector, which was not done in [5].

\section{Particle-Field Duality and Form-Factors}

In a quantum field theory with a spectrum of massive particles, one deals with the space of multiparticle states $\mathcal{H}_{\mathcal{P}}$. In the context of the integrable theories in 2 dimensions, we can describe $\mathcal{H}_{\mathcal{P}}$ as follows. Introduce the so-called Faddeev-Zamolodchikov operators $Z_{\epsilon}^{\dagger}(\theta), Z^{\epsilon}(\theta)$, where $\theta$ is the rapidity parameterizing the energy-momentum $(E=\mathrm{m} \cosh \theta, P=\mathrm{m} \sinh \theta)$, and $\epsilon$ is an isotopic index, satisfying:

$$
\begin{aligned}
& Z^{\epsilon_{1}}\left(\theta_{1}\right) Z^{\epsilon_{2}}\left(\theta_{2}\right)=S_{\epsilon_{1}^{\prime} \epsilon_{2}^{\prime}}^{\epsilon_{\epsilon_{2}}}\left(\theta_{12}\right) Z^{\epsilon_{2}^{\prime}}\left(\theta_{2}\right) Z^{\epsilon_{1}^{\prime}}\left(\theta_{1}\right) \\
& Z_{\epsilon_{1}}^{\dagger}\left(\theta_{1}\right) Z_{\epsilon_{2}}^{\dagger}\left(\theta_{2}\right)=S_{\epsilon_{1} \epsilon_{2}}^{\epsilon_{1}^{\prime} \epsilon_{2}^{\prime}}\left(\theta_{12}\right) Z_{\epsilon_{2}^{\prime}}^{\dagger}\left(\theta_{2}\right) Z_{\epsilon_{1}^{\prime}}^{\dagger}\left(\theta_{1}\right) \\
& Z^{\epsilon_{1}}\left(\theta_{1}\right) Z_{\epsilon_{2}}^{\dagger}\left(\theta_{2}\right)-S_{\epsilon_{2} \epsilon_{1}^{\prime}}^{\epsilon_{2}^{\prime} \epsilon_{1}}\left(\theta_{21}\right) Z_{\epsilon_{2}^{\prime}}^{\dagger}\left(\theta_{2}\right) Z^{\epsilon_{1}^{\prime}}\left(\theta_{1}\right)=\delta_{\epsilon_{2}}^{\epsilon_{1}} \delta\left(\theta_{1}-\theta_{2}\right) .
\end{aligned}
$$

Above, $S$ is the S-matrix, and $\theta_{12}=\theta_{1}-\theta_{2}$. Define particle states as follows:

$$
\begin{aligned}
& \left|\theta_{1} \cdots \theta_{n}\right\rangle_{\epsilon_{1} \cdots \epsilon_{n}}=Z_{\epsilon_{1}}^{\dagger}\left(\theta_{1}\right) \cdots Z_{\epsilon_{n}}^{\dagger}\left(\theta_{n}\right)|0\rangle \\
& \epsilon_{1} \cdots \epsilon_{n}\left\langle\theta_{1} \cdots \theta_{n}\right|=\langle 0| Z^{\epsilon_{1}}\left(\theta_{1}\right) \cdots Z^{\epsilon_{n}}\left(\theta_{n}\right) .
\end{aligned}
$$


The space $\mathcal{H}_{\mathcal{P}}$ and its dual $\mathcal{H}_{\mathcal{P}}^{*}$ are spanned by the above states:

$$
\begin{aligned}
\mathcal{H}_{\mathcal{P}} & =\left\{\oplus_{n}\left|\theta_{1} \cdots \theta_{n}\right\rangle_{\epsilon_{1} \cdots \epsilon_{n}}\right\} \\
\mathcal{H}_{\mathcal{P}}^{*} & =\left\{\oplus_{n}{ }^{\epsilon_{1} \cdots \epsilon_{n}}\left\langle\theta_{1} \cdots \theta_{n}\right|\right\} .
\end{aligned}
$$

In this space one has the completeness relation

$$
1=\sum_{\vec{\theta}}|\vec{\theta}\rangle\left\langle\overleftarrow{\theta}\left|=\sum_{n=0}^{\infty} \frac{1}{n !} \sum_{\left\{\epsilon_{i}\right\}} \int d \theta_{1} \cdots d \theta_{n}\right| \theta_{1}, \cdots, \theta_{n}\right\rangle_{\epsilon_{1} \cdots \epsilon_{n}}{ }^{\epsilon_{n} \cdots \epsilon_{1}}\left\langle\theta_{n}, \cdots, \theta_{1}\right|
$$

Next consider the space of fields $\mathcal{H}_{\mathcal{F}}$. Let $\mathcal{F}$ denote the complete space of fields, and define $\left|\Phi_{i}\right\rangle=\Phi_{i}(0)|0\rangle, \Phi_{i}(x) \in \mathcal{F}$. The space $\mathcal{H}_{\mathcal{F}}$ is defined as follows

$$
\mathcal{H}_{\mathcal{F}}=\left\{\oplus_{\Phi_{i} \in \mathcal{F}}\left|\Phi_{i}\right\rangle\right\}
$$

Form factors are matrix elements of fields in the space of states $\mathcal{H}_{\mathcal{P}}$. The basic form factors ${ }^{\epsilon_{1} \cdots \epsilon_{n}}\left\langle\theta_{1} \cdots \theta_{n} \mid \Phi\right\rangle$, from which the more general matrix elements may be obtained by crossing symmetry, are inner products of states in $\mathcal{H}_{\mathcal{F}}$ with states in $\mathcal{H}_{\mathcal{P}}^{*}$. The completeness relation (2.3) allows us to map states in $\mathcal{H}_{\mathcal{F}}$ to states in $\mathcal{H}_{\mathcal{P}}$, i.e. to view $|\Phi\rangle \in \mathcal{H}_{\mathcal{P}}$ :

$$
\left|\Phi_{i}\right\rangle=\sum_{\vec{\theta}}|\vec{\theta}\rangle\left\langle\overleftarrow{\theta} \mid \Phi_{i}\right\rangle
$$

The intuitive simplicity of the space $\mathcal{H}_{\mathcal{P}}$ is responsible for this conventional way of thinking about form factors.

We give now a dual description of the same form factors. Let us suppose that one can define a dual to the space of fields $\mathcal{H}_{\mathcal{F}}^{*}$ with inner product and completeness relation:

$$
\begin{aligned}
\left\langle\Phi^{i} \mid \Phi_{j}\right\rangle & =\delta_{j}^{i} \\
1 & =\sum_{i}\left|\Phi_{i}\right\rangle\left\langle\Phi^{i}\right| .
\end{aligned}
$$

Then one can map a state $|\vec{\theta}\rangle \in \mathcal{H}_{\mathcal{P}}$ into $\mathcal{H}_{\mathcal{F}}$. The dual statement is

$$
\epsilon_{1} \cdots \epsilon_{n}\left\langle\theta_{1} \cdots \theta_{n}\right|=\sum_{\Phi_{i} \in \mathcal{F}} \epsilon_{1} \cdots \epsilon_{n}\left\langle\theta_{1} \cdots \theta_{n} \mid \Phi_{i}\right\rangle\left\langle\Phi^{i}\right| \quad \in \mathcal{H}_{\mathcal{F}}^{*}
$$

In order to work the above simple remarks into an efficient means of computing form factors, one must work explicitly with the space $\mathcal{H}_{\mathcal{F}}$. The space $\mathcal{H}_{\mathcal{F}}$ diagonalizes the 
Lorentz boost operator $L$, thus it can be understood as the space of radial quantization [8] [5]. We remark that in conformal field theory [9] one deals precisely with the space of fields, and it was argued in [5] that for many massive quantum field theories, the space $\mathcal{H}_{\mathcal{F}}$ is identical to its description in the ultraviolet conformal field theory.

In order to use these ideas to compute form factors, we need to introduce the notion of vertex operators. The formula (2.7) implies that one can map states in $\mathcal{H}_{\mathcal{P}}^{*}$ to states in $\mathcal{H}_{\mathcal{F}}^{*}$. We call this map the 'particle-field map'. We construct this map explicitly by defining vertex operators $V^{\epsilon}(\theta)$ as follows:

$$
\epsilon_{1} \cdots \epsilon_{n}\left\langle\theta_{1} \cdots \theta_{n}\right|=\langle\Omega| V^{\epsilon_{1}}\left(\theta_{1}\right) \cdots V^{\epsilon_{n}}\left(\theta_{n}\right) \quad \in \mathcal{H}_{\mathcal{F}}^{*}
$$

where $\langle\Omega|$ is a fixed 'vacuum' state. The vertex operators are distinguished from the Faddeev-Zamolodchikov operators $Z(\theta)$ since they act on completely different spaces. However the basic algebraic relations satisfied by the $Z$ operators continue to be satisfied by the $V$ operators. The vertex operators $V^{\epsilon}(\theta)$ operate in the space $\mathcal{H}_{\mathcal{F}}$ :

$$
V^{\epsilon}(\theta): \quad \mathcal{H}_{\mathcal{F}} \rightarrow \mathcal{H}_{\mathcal{F}}
$$

In the sequel we will describe how to construct these vertex operators explicitly using radial quantization. Once the vertex operators are constructed, the form factors $\epsilon_{1} \cdots \epsilon_{n}\left\langle\theta_{1} \cdots \theta_{n} \mid \Phi_{i}\right\rangle$ are computed directly in the discrete space $\mathcal{H}_{\mathcal{F}}$ using (2.8).

We close this section with some remarks on Green's functions. One way to define Green's functions is to insert the identity (2.3) between fields in the matrix element, leading to an infinite integral representation. Just as for form factors, one can instead insert the identity in $\mathcal{H}_{\mathcal{F}}$. The fundamental object of interest is the following matrix element:

$$
C_{i j}^{k}(x)=\left\langle\Phi^{k}\left|\Phi_{i}(x)\right| \Phi_{j}\right\rangle
$$

These are equivalent to the operator product coefficients:

$$
\Phi_{i}(x) \Phi_{j}(0)=\sum_{k} C_{i j}^{k}(x) \Phi_{k}(0)
$$

Defining $\Phi_{0} \equiv 1$, one sees that $C_{i j}^{0}(x)=\left\langle 0\left|\Phi_{i}(x) \Phi_{j}(0)\right| 0\right\rangle$ are the 2-point functions. Multipoint functions can be expressed in terms of the above coefficients. Manifest translation invariance is lost in the resulting expressions, as one would expect from radial quantization. Though these structures provide a different point of view on the correlation functions, by themselves they do not appear to simplify the problem. 


\section{Radial Quantization}

We will consider the sine-Gordon theory, defined by the action

$$
S=\frac{1}{4 \pi} \int d^{2} z\left(\partial_{z} \phi \partial_{\bar{z}} \phi+4 \lambda \cos (\widehat{\beta} \phi)\right)
$$

at the free fermion point, which occurs at $\widehat{\beta}=1 \llbracket 10]$. The free Dirac fermion fields $\psi^{ \pm}, \bar{\psi}^{ \pm}$ carry $U(1)$ charge \pm 1 , and their dynamics is governed by the action

$$
S=-\frac{1}{4 \pi} \int d x d t\left(\bar{\psi}^{-} \partial_{z} \bar{\psi}^{+}+\psi^{-} \partial_{\bar{z}} \psi^{+}+i \mathrm{~m}\left(\psi^{-} \bar{\psi}^{+}-\bar{\psi}^{-} \psi^{+}\right)\right)
$$

with the equations of motion

$$
\partial_{z} \bar{\psi}^{ \pm}=i \mathrm{~m} \psi^{ \pm}, \quad \partial_{\bar{z}} \psi^{ \pm}=-i \mathrm{~m} \bar{\psi}^{ \pm}
$$

In conventional temporal quantization, the expansion of the fermion fields in terms of momentum space operators is as follows:

$$
\begin{aligned}
& \psi^{+}(x, t)=\sqrt{\mathrm{m}} \int_{-\infty}^{\infty} d \theta e^{\theta / 2}\left(Z^{+}(\theta) e^{-i p(\theta) \cdot x}-Z_{-}^{\dagger}(\theta) e^{i p(\theta) \cdot x}\right) \\
& \bar{\psi}^{+}(x, t)=-i \sqrt{\mathrm{m}} \int_{-\infty}^{\infty} d \theta e^{-\theta / 2}\left(Z^{+}(\theta) e^{-i p(\theta) \cdot x}+Z_{-}^{\dagger}(\theta) e^{i p(\theta) \cdot x}\right),
\end{aligned}
$$

with $\psi^{-}=\left(\psi^{+}\right)^{\dagger}, \bar{\psi}^{-}=\left(\bar{\psi}^{+}\right)^{\dagger}$, and $\left(Z^{\epsilon}\right)^{\dagger}=Z_{\epsilon}^{\dagger}$.

Consider now radial quantization of the free-fermion theory [8] [11] [12]. Define the usual polar coordinates as in (1.1). One can define two distinct sectors, the periodic (p) and anti-periodic (a), with expansions

$$
\Psi_{(a, p)}^{ \pm}=\left(\begin{array}{l}
\bar{\psi}^{ \pm} \\
\psi^{ \pm}
\end{array}\right)=\sum_{\omega}\left[b_{\omega}^{ \pm} \Psi_{-\omega-1 / 2}^{(a, p)}+\bar{b}_{\omega}^{ \pm} \bar{\Psi}_{-\omega-1 / 2}^{(a, p)}\right]
$$

where for the periodic sector $\omega \in Z+1 / 2$, and for the anti-periodic sector $\omega \in Z$. The basis spinors can be found as solutions to the equations of motion (3.3) in radial coordinates:

$$
\begin{aligned}
& \Psi_{-\omega-1 / 2}^{(a)}=\Gamma\left(\frac{1}{2}-\omega\right) \mathrm{m}^{\omega+1 / 2}\left(\begin{array}{c}
i e^{i\left(\frac{1}{2}-\omega\right) \varphi} I_{\frac{1}{2}-\omega}(\mathrm{m} r) \\
e^{-i\left(\omega+\frac{1}{2}\right) \varphi} I_{-\omega-\frac{1}{2}}(\mathrm{~m} r)
\end{array}\right) \\
& \bar{\Psi}_{-\omega-1 / 2}^{(a)}=\Gamma\left(\frac{1}{2}-\omega\right) \mathrm{m}^{\omega+1 / 2}\left(\begin{array}{c}
e^{i\left(\frac{1}{2}+\omega\right) \varphi} I_{-\frac{1}{2}-\omega}(\mathrm{m} r) \\
-i e^{-i\left(\frac{1}{2}-\omega\right) \varphi} I_{\frac{1}{2}-\omega}(\mathrm{m} r)
\end{array}\right),
\end{aligned}
$$




$$
\begin{aligned}
& \Psi_{-\omega-1 / 2}^{(p)}=\frac{2 \mathrm{~m}^{\omega+1 / 2}}{\Gamma\left(\frac{1}{2}+\omega\right)}\left(\begin{array}{c}
-i e^{i\left(\frac{1}{2}-\omega\right) \varphi} K_{\omega-\frac{1}{2}}(\mathrm{~m} r) \\
e^{-i\left(\omega+\frac{1}{2}\right) \varphi} K_{\omega+\frac{1}{2}}(\mathrm{~m} r)
\end{array}\right) \quad \omega \geq 1 / 2 \\
& \bar{\Psi}_{-\omega-1 / 2}^{(p)}=\frac{2 \mathrm{~m}^{\omega+1 / 2}}{\Gamma\left(\frac{1}{2}+\omega\right)}\left(\begin{array}{c}
e^{i\left(\frac{1}{2}+\omega\right) \varphi} K_{\omega+\frac{1}{2}}(\mathrm{~m} r) \\
i e^{i\left(\omega-\frac{1}{2}\right) \varphi} K_{\omega-\frac{1}{2}}(\mathrm{~m} r)
\end{array}\right) \quad \omega \geq 1 / 2,
\end{aligned}
$$

and $\Psi_{-\omega-1 / 2}^{(p)}, \bar{\Psi}_{-\omega-1 / 2}^{(p)}$ for $\omega \leq-1 / 2$ have the same expression as in the anti-periodic sector.

In the quantum theory these modes satisfy simple anti-commutation relations:

$$
\left\{b_{\omega}^{+}, b_{\omega^{\prime}}^{-}\right\}=\left\{\bar{b}_{\omega}^{+}, \bar{b}_{\omega^{\prime}}^{-}\right\}=\delta_{\omega,-\omega^{\prime}}, \quad\left\{b_{\omega}, \bar{b}_{\omega^{\prime}}\right\}=0 .
$$

These modes diagonalize the Lorentz boost operator:

$$
\left[L, b_{\omega}^{ \pm}\right]=-\omega b_{\omega}^{ \pm}, \quad\left[L, \bar{b}_{\omega}^{ \pm}\right]=\omega \bar{b}_{\omega}^{ \pm} .
$$

The space of radial quantization consists of Fock modules built from these oscillators. In the periodic sector, the vacuum is defined to satisfy

$$
b_{\omega}^{ \pm}|0\rangle=\bar{b}_{\omega}^{ \pm}|0\rangle=0, \quad \omega \geq 1 / 2 .
$$

The vacuum $|0\rangle$ is the physical one. One constructs Fock modules for the periodic sector as follows:

$$
\mathcal{H}_{p}^{L}=\left\{b_{-\omega_{1}}^{\epsilon_{1}} b_{-\omega_{2}}^{\epsilon_{2}} \cdots|0\rangle\right\}, \quad \mathcal{H}_{p}^{R}=\left\{\bar{b}_{-\omega_{1}}^{\epsilon_{1}} \bar{b}_{-\omega_{2}}^{\epsilon_{2}} \cdots|0\rangle\right\},
$$

where $\omega_{i} \in Z+1 / 2, \omega_{i} \geq 1 / 2$.

In the anti-periodic sector, due to the existence of the zero modes, the 'vacuum' states are doubly degenerate. These vacua $\left| \pm \frac{1}{2}\right\rangle_{L}$ and $\left| \pm \frac{1}{2}\right\rangle_{R}$ are characterized as follows:

$$
\begin{aligned}
& b_{0}^{ \pm}\left|\mp \frac{1}{2}\right\rangle_{L}=\left| \pm \frac{1}{2}\right\rangle_{L}, \quad b_{0}^{ \pm}\left| \pm \frac{1}{2}\right\rangle_{L}=0, \quad b_{n}^{ \pm}\left| \pm \frac{1}{2}\right\rangle_{L}=0, \quad n \geq 1 \\
& \bar{b}_{0}^{ \pm}\left|\mp \frac{1}{2}\right\rangle_{R}=\left| \pm \frac{1}{2}\right\rangle_{R}, \quad \bar{b}_{0}^{ \pm}\left| \pm \frac{1}{2}\right\rangle_{R}=0, \quad \bar{b}_{n}^{ \pm}\left| \pm \frac{1}{2}\right\rangle_{R}=0, \quad n \geq 1 .
\end{aligned}
$$

The dual vacua $\left\langle \pm \frac{1}{2}\right|$ are defined by the inner products

$$
{ }_{L}\left\langle\mp \frac{1}{2} \mid \pm \frac{1}{2}\right\rangle_{L}={ }_{R}\left\langle\mp \frac{1}{2} \mid \pm \frac{1}{2}\right\rangle_{R}=1 \text {. }
$$

The anti-periodic Fock spaces are defined as

$$
\mathcal{H}_{a_{ \pm}}^{L}=\left\{b_{-n_{1}}^{\epsilon_{1}} b_{-n_{2}}^{\epsilon_{2}} \cdots \cdot\left| \pm \frac{1}{2}\right\rangle_{L}\right\}, \quad \mathcal{H}_{a_{ \pm}}^{R}=\left\{\bar{b}_{-n_{1}}^{\epsilon_{1}} \bar{b}_{-n_{2}}^{\epsilon_{2}} \cdots \cdots\left| \pm \frac{1}{2}\right\rangle_{R}\right\}
$$


for $n_{i} \in Z \geq 1$.

The space of radial quantization corresponds precisely to the space of fields $\mathcal{H}_{\mathcal{F}}$ described in the last section. Namely, in the periodic sector $\mathcal{H}_{\mathcal{F}}^{(p)}=\mathcal{H}_{p}^{L} \otimes \mathcal{H}_{p}^{R}$, and in the anti-periodic sector $\mathcal{H}_{\mathcal{F}}^{(a)}=\mathcal{H}_{a}^{L} \otimes \mathcal{H}_{a}^{R}$, where $\mathcal{H}_{a}^{L, R}=\mathcal{H}_{a_{+}}^{L, R} \oplus \mathcal{H}_{a_{-}}^{L, R}$. What is remarkable about this result is that the structure of the space of fields is identical to that in the massless conformal limit. One finds that in the limit $r \rightarrow 0$, the Bessel functions in the expansions (3.5) behave in such a way that the mass-dependent terms in the expression $\Phi(r)|0\rangle$ disappear. Arguments explaining this phenomenon were given in [5].

We present some simple examples that we will use later. The $U(1)$ current $J_{\mu}$ has components $J_{z}=\psi^{+} \psi^{-}, J_{\bar{z}}=\bar{\psi}^{+} \bar{\psi}^{-}$. Also of interest is the energy-momentum tensor:1

$$
\begin{array}{ll}
T_{z z}=\frac{1}{2}\left(\psi^{-} \partial_{z} \psi^{+}-\partial_{z} \psi^{-} \psi^{+}\right), & T_{\overline{z z}}=\frac{1}{2}\left(\bar{\psi}^{-} \partial_{\bar{z}} \bar{\psi}^{+}-\partial_{\bar{z}} \bar{\psi}^{-} \bar{\psi}^{+}\right) \\
T_{z \bar{z}}=\frac{1}{2}\left(\psi^{-} \partial_{\bar{z}} \psi^{+}-\partial_{\bar{z}} \psi^{-} \psi^{+}\right), & T_{\bar{z} z}=\frac{1}{2}\left(\bar{\psi}^{-} \partial_{z} \bar{\psi}^{+}-\partial_{z} \bar{\psi}^{-} \bar{\psi}^{+}\right) .
\end{array}
$$

Using the expansions (3.5), the asymptotic expansions of the Bessel functions as $r \rightarrow 0$, and the properties of the vacuum (3.10), one finds

$$
\begin{aligned}
\partial_{z}^{n} \psi^{ \pm}(0)|0\rangle & =n ! b_{-n-\frac{1}{2}}^{ \pm}|0\rangle, \quad \partial_{\bar{z}}^{n} \bar{\psi}^{ \pm}(0)|0\rangle=n ! \bar{b}_{-n-\frac{1}{2}}^{ \pm}|0\rangle \\
J_{z}(0)|0\rangle & =b_{-\frac{1}{2}}^{+} b_{-\frac{1}{2}}^{-}|0\rangle, \quad J_{\bar{z}}(0)|0\rangle=\bar{b}_{-\frac{1}{2}}^{+} \bar{b}_{-\frac{1}{2}}^{-}|0\rangle \\
T_{z z}(0)|0\rangle & =\frac{1}{2}\left(b_{-\frac{1}{2}}^{-} b_{-\frac{3}{2}}^{+}-b_{-\frac{3}{2}}^{-} b_{-\frac{1}{2}}^{+}\right)|0\rangle, \quad T_{\overline{z z}}(0)|0\rangle=\frac{1}{2}\left(\bar{b}_{-\frac{1}{2}}^{-} \bar{b}_{-\frac{3}{2}}^{+}-\bar{b}_{-\frac{3}{2}}^{-} \bar{b}_{-\frac{1}{2}}^{+}\right)|0\rangle \\
T_{z \bar{z}}(0)|0\rangle & =T_{\bar{z} z}(0)|0\rangle=-\frac{i \mathrm{~m}}{2}\left(b_{-\frac{1}{2}}^{-} \bar{b}_{-\frac{1}{2}}^{+}-\bar{b}_{-\frac{1}{2}}^{-} b_{-\frac{1}{2}}^{+}\right)|0\rangle .
\end{aligned}
$$

The anti-periodic sector is more interesting, since here one can access fields in the sine-Gordon theory which are not simply expressed in terms of the fermion fields. By studying the operator product expansion, the following identification was made in [5]:

$$
e^{ \pm i \phi(0) / 2}|0\rangle=(\mathrm{cm})^{1 / 4}\left(\left| \pm \frac{1}{2}\right\rangle_{L} \otimes\left|\mp \frac{1}{2}\right\rangle_{R}\right) \equiv(\mathrm{cm})^{1 / 4}\left| \pm \frac{1}{2}\right\rangle,
$$

where $\phi(z, \bar{z})$ is the local SG field, and $c$ is an undetermined dimensionless constant. The mass dimension of $1 / 4$ on the RHS is fixed by the known scaling dimension of the fields $\exp ( \pm i \phi / 2)$, which is the same as in the conformal limit. (The constant $c$ can be fixed by specifying the 1-point functions of these fields; see below.) The fields $\exp ( \pm i \phi / 2)$ are nontrivial terms of the fermions, since the bosonization relation is $\cos (\phi)=\left(\psi^{-} \bar{\psi}^{+}-\bar{\psi}^{-} \psi^{+}\right)$. All other states in $\mathcal{H}_{\mathcal{F}}^{(a)}$ correspond to regularized products of fermion fields and their derivatives with the basic fields $\exp ( \pm i \phi / 2)$.

1 We normalize conserved currents such that $Q=\frac{1}{4 \pi} \int d x\left(J_{z}+J_{\bar{z}}\right)$ is the properly normalized conserved charge. 


\section{Particle-Field Maps}

The basic property that allows us to construct explicitly the particle-field maps of the form (2.8) is the fact that the space of radial quantization can be obtained from the usual space of temporal quantization by appropriate analytic continuation in momentum space. Since the situation in the periodic versus the anti-periodic sector is quite different, we treat them separately.

\subsection{Periodic Sector}

Let us combine the creation and annihilation operators of temporal quantization into a single operator as follows. Define the momentum space variable $u$ as

$$
u=e^{\theta}
$$

and define operators $\widehat{b}^{ \pm}(u)$ as

$$
\begin{array}{lrr}
\widehat{b}^{+}(u) & =2 \pi Z_{-}^{\dagger}(\theta), & \widehat{b}^{-}(u)=2 \pi Z_{+}^{\dagger}(\theta) \quad \text { for } u>0 \\
\widehat{b}^{+}(u) & =2 \pi i Z^{+}(\theta-i \pi), & \widehat{b}^{-}(u)=2 \pi i Z^{-}(\theta-i \pi) \quad \text { for } u<0 .
\end{array}
$$

Then the temporal quantization expansion (3.4) may be written as

$$
\Psi^{ \pm}=\left(\begin{array}{c}
\bar{\psi}^{ \pm} \\
\psi^{ \pm}
\end{array}\right)= \pm \sqrt{\mathrm{m}} \int_{-\infty}^{\infty} \frac{d u}{2 \pi i|u|} \widehat{b}^{ \pm}(u)\left(\begin{array}{c}
1 / \sqrt{u} \\
-i \sqrt{u}
\end{array}\right) e^{\mathrm{m} z u+\mathrm{m} \bar{z} / u}
$$

Let us now define the following prescription for analytic continuation of the $u$-integral in $(4.3)$ :

$$
\begin{aligned}
& \int_{0}^{\infty} \frac{d u}{2 \pi i|u|} \widehat{b}^{ \pm}(u) \rightarrow \oint_{\mathcal{C}_{<}} \frac{d u}{2 \pi i u}\left(b_{<}^{ \pm}(u)+\bar{b}_{<}^{ \pm}(u)\right) \\
& \int_{-\infty}^{0} \frac{d u}{2 \pi i|u|} \widehat{b}^{ \pm}(u) \rightarrow \int_{\mathcal{C}_{>}} \frac{d u}{2 \pi i u}\left(b_{>}^{ \pm}(u)+\bar{b}_{>}^{ \pm}(u)\right),
\end{aligned}
$$

where the contour $\mathcal{C}_{<}$is defined to be a closed contour on the unit circle in the complex $u$-plane, whereas $\mathcal{C}_{>}$runs from 0 to $\infty$ along a ray at an angle $\varphi$ above the negative $x$-axis in the $u$ plane. The operators on the RHS are defined to have the following expansions:

$$
\begin{array}{ll}
b_{<}^{ \pm}(u)= \pm i \sum_{\omega \leq-1 / 2} \Gamma\left(\frac{1}{2}-\omega\right) \mathrm{m}^{\omega} b_{\omega}^{ \pm} u^{\omega}, & b_{>}^{ \pm}(u)= \pm \sum_{\omega \geq 1 / 2} \frac{2 \pi(-1)^{\omega+1 / 2}}{\Gamma\left(\omega+\frac{1}{2}\right)} \mathrm{m}^{\omega} b_{\omega}^{ \pm} u^{\omega} \\
\bar{b}_{<}^{ \pm}(u)= \pm \sum_{\omega \leq-1 / 2} \Gamma\left(\frac{1}{2}-\omega\right) \mathrm{m}^{\omega} \bar{b}_{\omega}^{ \pm} u^{-\omega}, & \bar{b}_{>}^{ \pm}(u)= \pm i \sum_{\omega \geq 1 / 2} \frac{2 \pi(-1)^{\omega-1 / 2}}{\Gamma\left(\omega+\frac{1}{2}\right)} \mathrm{m}^{\omega} \bar{b}_{\omega}^{ \pm} u^{-\omega} .
\end{array}
$$


One can show, using well-known integral representations for the Bessel functions, that the analytic continuation (4.4), (4.5) of (4.3) yields the expansion (3.5) of radial quantization. (See [5].)

Now, consider the states

$$
\epsilon_{1} \cdots \epsilon_{n}\left\langle\theta_{1} \cdots \theta_{n}\right|=\frac{1}{(2 \pi i)^{n}}\langle 0| \widehat{b}^{\epsilon_{1}}\left(e^{-i \pi} u_{1}\right) \cdots \widehat{b}^{\epsilon_{n}}\left(e^{-i \pi} u_{n}\right), \quad u_{i}<0 .
$$

Using the analytic continuation (4.4), one can map the above state into the space of radial quantization. Bearing in mind that $u_{i}<0$ in (4.6), one uses the second formula in (4.4) to obtain the equation (2.8), where the vacuum and vertex operators are:

$$
\begin{aligned}
\langle\Omega| & =\langle 0| \\
V^{\epsilon}(\theta) & =\frac{1}{2 \pi i}\left(b_{>}^{\epsilon}\left(e^{-i \pi} u\right)+\bar{b}_{>}^{\epsilon}\left(e^{-i \pi} u\right)\right) .
\end{aligned}
$$

One can easily check the validity of (4.7) by verifying that it gives the correct form factors in some simple cases. As explained above, any field $\Phi$ in the periodic sector corresponds to a state $|\Phi\rangle$ in the space $\mathcal{H}_{\mathcal{F}}^{(p)}$ described in section 3 , and the form-factor is computed as

$$
\epsilon_{1} \cdots \epsilon_{n}\left\langle\theta_{1} \cdots \theta_{n} \mid \Phi\right\rangle=\left\langle 0\left|V^{\epsilon_{1}}\left(\theta_{1}\right) \cdots V^{\epsilon_{n}}\left(\theta_{n}\right)\right| \Phi\right\rangle
$$

Note that since only the radial annihilation operators appear in (4.7), all of the form factors in the periodic sector have the 'free field' property: for a given field, the $n$-particle form factors are all zero except for $n=n_{\psi}$, where $n_{\psi}$ is the number of free fermion fields needed to construct the field. Using (4.8), (4.7), and (3.16) one finds

$$
\begin{aligned}
\pm\left\langle\theta\left|\psi^{\mp}(0)\right| 0\right\rangle & = \pm \sqrt{\mathrm{m} u}, \quad \pm\left\langle\theta\left|\bar{\psi}^{\mp}(0)\right| 0\right\rangle= \pm i \sqrt{\mathrm{m} / u} \\
+-\left\langle\theta_{1}, \theta_{2}\left|J_{z}(0)\right| 0\right\rangle & =-\mathrm{m}\left(u_{1} u_{2}\right)^{1 / 2}, \quad+-\left\langle\theta_{1}, \theta_{2}\left|J_{\bar{z}}(0)\right| 0\right\rangle=\mathrm{m}\left(u_{1} u_{2}\right)^{-1 / 2} \\
+-\left\langle\theta_{1}, \theta_{2}\left|T_{z z}(0)\right| 0\right\rangle & =\frac{\mathrm{m}^{2}}{2}\left(\left(u_{1}\right)^{1 / 2}\left(u_{2}\right)^{3 / 2}-\left(u_{1}\right)^{3 / 2}\left(u_{2}\right)^{1 / 2}\right) \\
+-\left\langle\theta_{1}, \theta_{2}\left|T_{\overline{z z}}(0)\right| 0\right\rangle & =\frac{\mathrm{m}^{2}}{2}\left(\left(u_{1}\right)^{-3 / 2}\left(u_{2}\right)^{-1 / 2}-\left(u_{1}\right)^{-1 / 2}\left(u_{2}\right)^{-3 / 2}\right) \\
+-\left\langle\theta_{1}, \theta_{2}\left|T_{z \bar{z}}(0)\right| 0\right\rangle & =\frac{\mathrm{m}^{2}}{2}\left(\left(\frac{u_{1}}{u_{2}}\right)^{1 / 2}-\left(\frac{u_{2}}{u_{1}}\right)^{1 / 2}\right) .
\end{aligned}
$$

All the higher multiparticle form factors are zero for these fields, since they are all fermion bilinears. These form factors agree with expressions derived by the standard methods in the space of particles $\mathcal{H}_{\mathcal{P}}$. 


\subsection{Anti-Periodic Sector}

In this sector, the analytic continuation of (4.3) that reproduces the radial expansion (3.5) is the following:

$$
\int_{-\infty}^{\infty} \frac{d u}{2 \pi i|u|} \widehat{b}^{ \pm}(u) \rightarrow\left(\int_{\mathcal{C}_{\varphi}^{L}} \frac{d u}{2 \pi i u} b_{ \pm}(u)+\int_{\mathcal{C}_{\varphi}^{R}} \frac{d u}{2 \pi i u} \bar{b}_{ \pm}(u)\right)
$$

where $\mathcal{C}_{\varphi}^{L}, \mathcal{C}_{\varphi}^{R}$ are contours depending on the angular direction $\varphi$ of the cut displayed in figures 1,2 , and

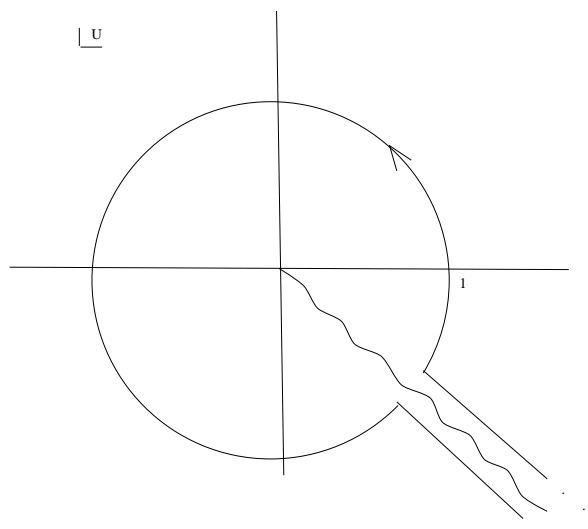

Figure 1. The contour $\mathcal{C}_{\varphi}^{L}$. The cut (wavy line) is oriented at an angle $\varphi$ from the negative $y$-axis. The circle is at $|u|=1$.

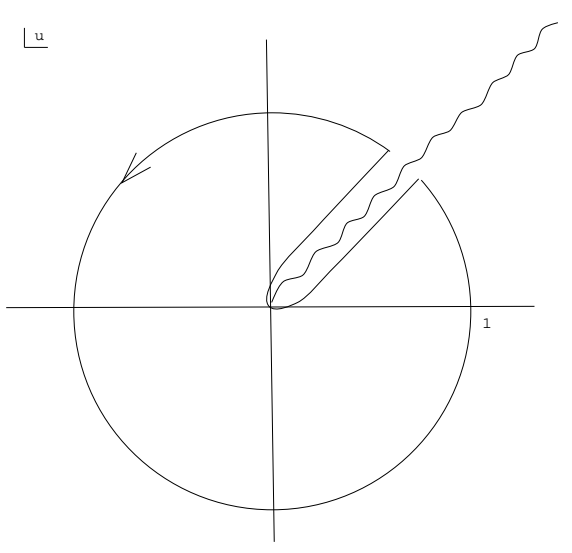

Figure 2. The contour $\mathcal{C}_{\varphi}^{R}$. The cut is oriented at an angle $\varphi$ from the positive $y$-axis. The circle is at $|u|=1$. 


$$
\begin{aligned}
& b^{ \pm}(u)= \pm i \sum_{\omega \in Z} \Gamma\left(\frac{1}{2}-\omega\right) \mathrm{m}^{\omega} b_{\omega}^{ \pm} u^{\omega} \\
& \bar{b}^{ \pm}(u)= \pm \sum_{\omega \in Z} \Gamma\left(\frac{1}{2}-\omega\right) \mathrm{m}^{\omega} \bar{b}_{\omega}^{ \pm} u^{-\omega}
\end{aligned}
$$

The most significant difference between the anti-periodic and periodic sectors is that here the analytic continuation (4.10) does not separate the radial creation operators from the annihilation operators, unlike in (4.4). This simple fact is what is responsible for the non-free properties of the form factors in this sector.

Repeating the reasoning given above for the periodic sector, we propose that again the formula $(2.8)$ is valid, where now

$$
\begin{aligned}
\langle\Omega| & =\left\langle\frac{1}{2}\right|+\left\langle-\frac{1}{2}\right| \\
V^{\epsilon}(\theta) & =\frac{1}{\sqrt{2 \pi^{2} i}}\left(b^{\epsilon}\left(e^{-i \pi} u\right)+\bar{b}^{\epsilon}\left(e^{-i \pi} u\right)\right) .
\end{aligned}
$$

The normalization of the vertex operators was chosen to satisfy the residue property

$$
V^{+}(\theta) V^{-}(\theta+\beta+i \pi) \sim \frac{1}{i \pi \beta}
$$

which leads to the proper residue axiom for the multiparticle form factors. From (3.17), one sees that the choice (4.12) for $\langle\Omega|$ is equivalent to the following vacuum expectation values:

$$
\left\langle 0\left|e^{ \pm i \phi(0) / 2}\right| 0\right\rangle=(\mathrm{cm})^{1 / 4}\left\langle\Omega \mid \pm \frac{1}{2}\right\rangle=(\mathrm{cm})^{1 / 4}
$$

One can use the above construction to compute the form factors of the fields $\exp ( \pm i \phi / 2)[5]$. For these fields, all of the form factors with a $U(1)$ neutral combination of an even number of particles is non-zero. The result is

$$
\begin{aligned}
+++\ldots---\cdots & \left\langle\theta_{1}, \theta_{2}, \cdots, \theta_{2 n}\left|e^{ \pm i \phi(0) / 2}\right| 0\right\rangle \\
= & (c \mathrm{~m})^{1 / 4}\left\langle\mp \frac{1}{2}\left|V^{+}\left(u_{1}\right) \cdots V^{+}\left(u_{n}\right) V^{-}\left(u_{n+1}\right) \cdots V^{-}\left(u_{2 n}\right)\right| \pm \frac{1}{2}\right\rangle \\
= & (c \mathrm{~m})^{1 / 4} \frac{( \pm 1)^{n}}{(i \pi)^{n}}(-1)^{n(n-1) / 2} \sqrt{u_{1} \cdots u_{2 n}}\left(\prod_{i=1}^{n}\left(\frac{u_{i+n}}{u_{i}}\right)^{ \pm 1 / 2}\right)\left(\prod_{i<j \leq n}\left(u_{i}-u_{j}\right)\right) \\
& \times\left(\prod_{n+1 \leq i<j}\left(u_{i}-u_{j}\right)\right)\left(\prod_{r=1}^{n} \prod_{s=n+1}^{2 n} \frac{1}{u_{r}+u_{s}}\right) .
\end{aligned}
$$


The above computation can be done using the Wick theorem with the 2-point functions

$$
\begin{aligned}
& { }_{L}\left\langle-\frac{1}{2}\left|b^{+}(u) b^{-}\left(u^{\prime}\right)\right|+\frac{1}{2}\right\rangle_{L}={ }_{R}\left\langle+\frac{1}{2}\left|\bar{b}^{+}(u) \bar{b}^{-}\left(u^{\prime}\right)\right|-\frac{1}{2}\right\rangle_{R}=\pi \frac{u^{\prime}}{u+u^{\prime}} \\
& { }_{L}\left\langle+\frac{1}{2}\left|b^{+}(u) b^{-}\left(u^{\prime}\right)\right|-\frac{1}{2}\right\rangle_{L}={ }_{R}\left\langle-\frac{1}{2}\left|\bar{b}^{+}(u) \bar{b}^{-}\left(u^{\prime}\right)\right|+\frac{1}{2}\right\rangle_{R}=-\pi \frac{u}{u+u^{\prime}} .
\end{aligned}
$$

However the computation is more easily done using the bosonization techniques of the next section. After some algebraic manipulation, one can see that these expressions agree with the known results, though they were originally computed using rather different methods 13 [3.

\section{Bosonization in Momentum Space}

Bosonization is a well-known construction in position space, and finds its most precise statement in conformal field theory. In this section, we will construct an exact bosonization in momentum space for the massive theory we are considering.

Though position space conformal bosonization is not physically relevant here, we review it in order to simply present a mathematical result that will facilitate understanding of the results presented below. Given a set of fermionic oscillators satisfying $\left\{c_{\omega}^{+}, c_{\omega^{\prime}}^{-}\right\}=$ $\delta_{\omega+\omega^{\prime}, 0}$, define a bosonic operator as follows:

$$
\rho_{n}=\mathrm{m}^{n} \sum_{\omega}: c_{n-\omega}^{+} c_{\omega}^{-}:
$$

These operators satisfy an infinite Heisenberg algebra:

$$
\left[\rho_{n}, \rho_{n^{\prime}}\right]=n \delta_{n+n^{\prime}, 0}
$$

Bosonization amounts to using the $\rho_{n}$ to construct a generating function for the $c_{\omega}^{ \pm}$as follows. Define a field

$$
-i \rho(z)=\sum_{n \neq 0} \rho_{n} \frac{z^{-n}}{n}-\rho_{0} \log (z)-\widetilde{\rho}_{0}
$$

where $\widetilde{\rho}_{0}$ is defined to satisfy

$$
\left[\rho_{0}, \widetilde{\rho}_{0}\right]=1
$$

2 The overall numerical factors in (4.15) differ from the ones in 3] however they agree with results implicit in [13]. Here the correct normalization is fixed by the residue property (4.13). 
Then one has

$$
c^{ \pm}(z)=\sum_{\omega} c_{\omega}^{ \pm} z^{-\omega-1 / 2}=: e^{ \pm i \rho(z)}:
$$

In conformal field theory, the above statements correspond to the exact bosonization of a free massless fermion, where $z$ is the left-moving light-cone coordinate. (See e.g. [14.) We now turn to the massive theory.

\subsection{Anti-Periodic Sector}

As shown in [5], in the massive theory one can use the constants of motion to formulate an exact bosonization. We review this here in order to compare with the periodic sector. In radial quantization, define the inner product of two spinors $A=\left(\begin{array}{c}\bar{a} \\ a\end{array}\right), B=\left(\begin{array}{l}\bar{b} \\ b\end{array}\right)$ as

$$
(A, B)=\frac{1}{4 \pi} \int_{-\pi}^{\pi} r d \varphi\left(e^{i \varphi} a b+e^{-i \varphi} \bar{a} \bar{b}\right)
$$

The conserved charges constructed in 15] [5 can all be expressed using the above inner product:

$$
\begin{aligned}
& Q_{-n}^{ \pm}=\frac{1}{2}\left(\Psi^{ \pm}, \partial_{z}^{n} \Psi^{ \pm}\right), \quad Q_{n}^{ \pm}=\frac{1}{2}\left(\Psi^{ \pm}, \partial \frac{n}{z} \Psi^{ \pm}\right) \\
& \alpha_{-n}=(-)^{n}\left(\Psi^{+}, \partial_{z}^{n} \Psi^{-}\right), \quad \alpha_{n}=(-)^{n}:\left(\Psi^{+}, \partial_{\bar{z}}^{n} \Psi^{-}\right):,
\end{aligned}
$$

where $n \geq 0$ is an integer, and for $Q_{n}^{ \pm}, n$ is odd. The operators $\left\{Q_{n}^{ \pm}, \alpha_{m}, m\right.$ even $\}$ generate a twisted affine $\widehat{s l(2)}$ algebra at level $k=0$, and the $\alpha_{n}$ for $n$ odd are the usual infinity of integrals of motion at odd integer Lorentz spin. In radial quantization, using Wronskian identities for the Bessel functions, one finds that the above conserved charges split into Left and Right pieces:

$$
Q_{n}^{ \pm}=Q_{n}^{ \pm, L}+Q_{-n}^{ \pm, R}, \quad \alpha_{n}=\alpha_{n}^{L}+\alpha_{-n}^{R}
$$

Both the $\mathrm{L}$ and the $\mathrm{R}$ pieces of the $\widehat{s l(2)}$ algebra separately satisfy a level $k=1$ algebra in the anti-periodic sector, and a level $k=0$ algebra in the periodic sector [5]. Here we are mainly interested in the $\alpha_{n}^{L, R}$ which have the explicit expressions:

$$
\alpha_{n}^{L}=\mathrm{m}^{|n|+n} \sum_{\omega \in S_{n}^{(a, p)}} \frac{\Gamma\left(\frac{1}{2}+\omega-n\right)}{\Gamma\left(\frac{1}{2}+\omega\right)}: b_{n-\omega}^{+} b_{\omega}^{-}:
$$

where the sums over $\omega$ run over $S_{n}^{(p)}\left(S_{n}^{(a)}\right)$ for the periodic (anti-periodic) sector, and

$$
S_{n}^{(a)}=Z, \quad \forall n, \quad S_{n}^{(p)}=\{\omega \in Z+1 / 2:|\omega-n / 2|>|n| / 2\} .
$$


Identical expressions apply to $\alpha_{n}^{R}$ with $b_{\omega}^{ \pm} \rightarrow \bar{b}_{\omega}^{ \pm}$.

In the anti-periodic sector, since $\alpha_{n}^{L, R}$ satisfy two separate Heisenberg algebras, they can be used to construct a bosonization. Define

$$
\rho_{n}=\mathrm{m}^{-|n|} \alpha_{n}^{L}, \quad \bar{\rho}_{n}=\mathrm{m}^{-|n|} \alpha_{n}^{R}
$$

satisfying (5.6) and $\left[\bar{\rho}_{n}, \bar{\rho}_{n^{\prime}}\right]=n \delta_{n+n^{\prime}, 0}$, and define the momentum space fields (recall $\left.u=e^{\theta}\right)$ :

$$
\begin{aligned}
& -i \rho(u)=\sum_{n \neq 0} \rho_{n} \frac{u^{n}}{n}+\rho_{0} \log (u)-\widetilde{\rho}_{0} \\
& -i \bar{\rho}(u)=\sum_{n \neq 0} \bar{\rho}_{n} \frac{u^{-n}}{n}-\bar{\rho}_{0} \log (u)-\widetilde{\bar{\rho}}_{0}
\end{aligned}
$$

where one also has $\left[\bar{\rho}_{0}, \widetilde{\bar{\rho}}_{0}\right]=1$. We further define an auxiliary vacuum satisfying

$$
\alpha_{n}^{L}|\emptyset\rangle=\alpha_{n}^{R}|\emptyset\rangle=0, \quad n \geq 0 ; \quad \widetilde{\alpha}_{0}^{L}|\emptyset\rangle, \quad \widetilde{\alpha}_{0}^{R}|\emptyset\rangle \neq 0 .
$$

This vacuum $|\emptyset\rangle$ is not to be confused with the physical vacuum $|0\rangle$ which resides in the periodic sector. One has the following expectation values:

$$
\begin{aligned}
\left\langle\emptyset\left|\rho(u) \rho\left(u^{\prime}\right)\right| \emptyset\right\rangle & =-\log \left(1 / u-1 / u^{\prime}\right) \\
\left\langle\emptyset\left|\bar{\rho}(u) \bar{\rho}\left(u^{\prime}\right)\right| \emptyset\right\rangle & =-\log \left(u-u^{\prime}\right) \\
\left\langle\emptyset\left|\prod_{i} e^{i \alpha_{i} \rho\left(u_{i}\right)}\right| \emptyset\right\rangle & =\prod_{i<j}\left(1 / u_{i}-1 / u_{j}\right)^{\alpha_{i} \alpha_{j}} \\
\left\langle\emptyset\left|\prod_{i} e^{i \alpha_{i} \bar{\rho}\left(u_{i}\right)}\right| \emptyset\right\rangle & =\prod_{i<j}\left(u_{i}-u_{j}\right)^{\alpha_{i} \alpha_{j}} .
\end{aligned}
$$

The bosonized expressions for the operators $b^{ \pm}(u), \bar{b}^{ \pm}(u)$ and the states $\left| \pm \frac{1}{2}\right\rangle$ follow from the basic commutation relations

$$
\begin{aligned}
& {\left[\alpha_{n}^{L}, b^{ \pm}(u)\right]=( \pm 1)^{n+1} \mathrm{~m}^{|n|} u^{-n} b^{ \pm}(u)} \\
& {\left[\alpha_{n}^{R}, \bar{b}^{ \pm}(u)\right]=( \pm 1)^{n+1} \mathrm{~m}^{|n|} u^{n} \bar{b}^{ \pm}(u)}
\end{aligned}
$$

and the 2-point functions (4.16). The commutation relations (5.15) are fundamental in the sense that they describe how the conserved charges are represented on asymptotic multiparticle states. One finds

$$
\frac{1}{\sqrt{ \pm \pi u}} b^{ \pm}(u)=: e^{ \pm i \rho( \pm u)}:, \quad \frac{ \pm 1}{\sqrt{ \pm \pi u}} \bar{b}^{ \pm}(u)=: e^{ \pm i \bar{\rho}( \pm u)}:
$$


where $-u=e^{-i \pi} u$, and

$$
\begin{aligned}
\left| \pm \frac{1}{2}\right\rangle_{L} & =: e^{ \pm i \rho(\infty) / 2}:|\emptyset\rangle_{L}, & \left| \pm \frac{1}{2}\right\rangle_{R}=: e^{ \pm i \bar{\rho}(0) / 2}:|\emptyset\rangle_{R} \\
{ }_{L}\left\langle \pm \frac{1}{2}\right| & =\lim _{u \rightarrow 0} u^{-1 / 4}\langle\emptyset|: e^{ \pm i \rho(u) / 2}:, & { }_{R}\left\langle \pm \frac{1}{2}\right|=\lim _{u \rightarrow \infty} u^{1 / 4}\langle\emptyset|: e^{ \pm i \bar{\rho}(u) / 2}: .
\end{aligned}
$$

One can easily check that this construction reproduces the form factors (4.15).

The above bosonization is mathematically identical to the one described in (5.1)(5.5). One can see this explicitly by making the canonical transformation (by 'canonical', we mean one that preserves the commutation relations):

$$
c_{\omega}^{ \pm}=\frac{( \pm 1)^{\omega}}{\sqrt{\pi}} \Gamma\left(\frac{1}{2}-\omega\right) b_{\omega}^{ \pm}, \quad \bar{c}_{\omega}^{ \pm}=\frac{( \pm 1)^{\omega}}{\sqrt{\pi}} \Gamma\left(\frac{1}{2}-\omega\right) \bar{b}_{\omega}^{ \pm} .
$$

\subsection{Periodic Sector}

In the periodic sector, one cannot use the constants of the motion $\alpha_{n}^{L, R}$ to construct a bosonization, due to the fact that in this sector: $\left[\alpha_{n}^{L}, \alpha_{m}^{R}\right]=\left[\alpha_{n}^{R}, \alpha_{m}^{R}\right]=0$. This can be traced to the gaps in the sets $S_{n}^{(p)}$. As explained in [5], this had to be the case for the following reason: the physical vacuum $|0\rangle$ is only invariant with respect to the conserved charges $\alpha_{n}$ if $\alpha_{n}^{L}$ and $\alpha_{n}^{R}$ separately commute.

One can still construct a bosonization in this sector modeled after the formulas (5.1)(5.5). Namely define the canonical transformation

$$
\begin{array}{ll}
c_{\omega}^{ \pm}=\frac{\Gamma\left(\frac{1}{2}-\omega\right)}{\sqrt{2 \pi}} b_{\omega}^{ \pm}, & \bar{c}_{\omega}^{ \pm}=\frac{\Gamma\left(\frac{1}{2}-\omega\right)}{\sqrt{2 \pi}} \bar{b}_{\omega}^{ \pm} \quad \text { for } \omega \leq-1 / 2 \\
c_{\omega}^{ \pm}=\frac{\sqrt{2 \pi}}{\Gamma\left(\frac{1}{2}+\omega\right)} b_{\omega}^{ \pm}, & \bar{c}_{\omega}^{ \pm}=\frac{\sqrt{2 \pi}}{\Gamma\left(\frac{1}{2}-\omega\right)} \bar{b}_{\omega}^{ \pm} \quad \text { for } \omega \geq 1 / 2,
\end{array}
$$

and define $\rho_{n}, \bar{\rho}_{n}$ as in (5.1), and $\rho(u), \bar{\rho}(u)$ as in (5.11). Then one has the bosonization formulas

$$
\begin{gathered}
\pm \frac{1}{\sqrt{2 \pi}}\left(\sqrt{-u} b_{>}^{ \pm}(-u)-i \sqrt{u} b_{<}^{ \pm}(u)\right)=: e^{ \pm i \rho(u)}: \\
\pm \frac{i}{\sqrt{2 \pi}}\left(\frac{1}{\sqrt{-u}} \bar{b}_{>}^{ \pm}(-u)-\frac{i}{\sqrt{u}} b_{<}^{ \pm}(u)\right)=: e^{ \pm i \bar{\rho}(u)}:
\end{gathered}
$$

\section{Conclusions}

Though we have limited ourselves to perhaps the simplest possible case of the freefermion point of the sine-Gordon theory, we believe the ideas presented here can lead to a new framework for computing form factors in massive integrable quantum field theory. In 
this approach, since a complete description of the space of fields $\mathcal{H}_{\mathcal{F}}$ is provided from the outset via radial quantization, the complete set of solutions to the form factor bootstrap is automatically yielded. In the bosonized construction in section 5, an important role was played by the affine $\widehat{s l(2)}$ symmetry. Since away from the free-fermion point this symmetry is deformed to a $\mathcal{U}_{q}(\widehat{s l(2)})$ symmetry [16], with $q=\exp \left(-2 \pi i / \widehat{\beta}^{2}\right)$, this quantum affine symmetry is expected to be important for the general construction. The results contained in [17] 18] [19] should prove useful.

Readers with some familiarity with conformal field theory will doubtless see the strong parallels of this subject with the work presented here. For the example we have developed, we have shown that in radial quantization form factors can be computed as correlation functions in momentum space, and these correlation functions are very similar in structure to conformal spacetime correlation functions. Furthermore, for the purposes of computing form factors, one can describe the space of fields in the same way as is done in the ultraviolet conformal field theory. In a definite sense, we have shown that starting from a description of the space of fields in a conformal field theory and the basic operators from which one constructs this space (in our case, we mean the operators $b_{\omega}^{ \pm}, \bar{b}_{\omega}^{ \pm}$), then one can reconstruct a massive theory and its form factors by constructing the vertex operators. It is important to understand if this is possible more generally.

\section{Acknowledgements}

We would like to thank Denis Bernard and Sergei Lukyanov for discussions. This work is supported by an Alfred P. Sloan Foundation fellowship, and the National Science Foundation in part through the National Young Investigator program. 


\section{References}

[1] A. B. Zamolodchikov and Al. B. Zamolodchikov, Annals Phys. 120 (1979) 253.

[2] M. Karowski and P. Weisz, Nucl. Phys. B139 (1978) 445.

[3] F. A. Smirnov, Form Factors in Completely Integrable Models of Quantum Field Theory, in Advanced Series in Mathematical Physics 14, World Scientific, 1992.

[4] F. A. Smirnov, J. Phys. A: Math. Gen. 19 (1986) L575.

[5] A. LeClair, Spectrum Generating Affine Lie Algebras in Massive Field Theory, hepth/9305110, to appear in Nucl. Phys. B.

[6] S. Lukyanov, Free Field Representation for Massive Integrable Models, and Correlators of the Jost Functions in the Sine-Gordon Model, Rutgers preprints RU-93-30, RU-9355 .

[7] B. Davies, O. Foda, M. Jimbo, T. Miwa and A. Nakayashiki, Commun. Math. Phys. 151 (1993) 89; M. Jimbo, K. Miki, T. Miwa and A. Nakayashiki, Phys. Lett. A168 (1992) 256.

[8] S. Fubini, A. J. Hanson, and R. Jackiw, Phys. Rev. D7 (1973) 1732.

[9] A. A. Belavin, A. M. Polyakov, and A. B. Zamolodchikov, Nucl. Phys. B241 (1984) 333.

[10] S. Coleman, Phys. Rev. D 11 (1975) 2088.

[11] H. Itoyama and H. B. Thacker, Nucl. Phys. B320 (1989) 541.

[12] P. Griffin, Nucl. Phys. B334, 637.

[13] B. Schroer and T. T. Truong, Nucl. Phys. B144 (1978) 80 ;

E. C. Marino, B. Schroer, and J. A. Swieca, Nucl. Phys. B200 (1982) 473.

[14] P. Ginsparg, Les Houches Lectures 1988, in Fields, Strings and Critical Phenomena, E. Brézin and J. Zinn-Justin Eds., North Holland (1990).

[15] E. Abdalla, M. C. B. Abdalla, G. Sotkov, and M. Stanishkov, Off Critical Current Algebras, Univ. Sao Paulo preprint, IFUSP-preprint-1027, Jan. 1993.

[16] D. Bernard and A. LeClair, Commun. Math. Phys. 142 (1991) 99; Phys. Lett. B247 (1990) 309.

[17] I. B. Frenkel and N. Yu. Reshetikhin, Commun. Math. Phys. 146 (1992) 1.

[18] F. A. Smirnov, Int. J. Mod. Phys. A7, Suppl. 1B (1992) 813.

[19] I. B. Frenkel and N. Jing, Proc. Natl. Acad. Sci. USA 85 (1988) 9373. 